\title{
Echinacoside suppresses pancreatic adenocarcinoma cell growth by inducing apoptosis via the mitogen-activated protein kinase pathway
}

\author{
WEI WANG, JINBIN LUO, YINGHUI LIANG and XINFENG LI
}

Department of General Surgery, Second Affiliated Hospital of Fujian Medical University, Quanzhou, Fujian 362000, P.R. China

Received December 14, 2014; Accepted August 24, 2015

DOI: $10.3892 / \mathrm{mmr} .2016 .4867$

\begin{abstract}
The clinical application of natural products derived from traditional Chinese medicine has gained attention in cancer chemotherapeutics. Echinacoside (ECH), one of the phenylethanoids, isolated from the stems of Cistanches salsa (a Chinese herbal medicine) has tissue-protective and anti-apoptotic effects on the central nervous system. However, it remains largely elusive whether ECH possesses tumor suppressive activity. In the present study, it was demonstrated that $\mathrm{ECH}$ can markedly inhibit the proliferation of pancreatic adenocarcinoma cells by inducing the production of reactive oxygen species and the perturbation of mitochondrial membrane potential and thus triggering apoptosis. Furthermore, it was elucidated that ECH represses tumor cell growth through modulating MAPK activity. In conclusion, this study reveals an novel function of $\mathrm{ECH}$ in preventing cancer development, and implies that the usage of ECH could be a potential chemotherapeutic strategy for cancer.
\end{abstract}

\section{Introduction}

Cancer is a life-threatening disease and one of the leading causes of mortality worldwide. Cancer cells are characterized by inhibited apoptosis, uncontrolled growth and proliferation, and metastasis (1). The development of cancer, often initiated by genetic alterations, is a complex process that involves interactions between oncogenic proteins and tumor suppressors forming an intricate signaling network. For example, the oncogene $M y c$ has been found to be amplified or overexpressed in various types of cancer and the $M y c$-encoded oncoprotein can promote tumorigenesis by driving cell cycle progression, stimulating cell growth and inducing angiogenesis (2). However, the Myc-triggered oncogenic signals can activate

Correspondence to: Dr Xinfeng Li, Department of General Surgery, Second Affiliated Hospital of Fujian Medical University, 34 North Zhongshan Road, Quanzhou, Fujian 362000, P.R. China

E-mail: dzbmw022@126.com

Key words: echinacoside, traditional Chinese medicine, apoptosis, reactive oxygen species, mitochondrial membrane potential the p53 tumor suppressor pathway via the induction of the tumor suppressor, ARF that mitigates the oncogenic E3-ligase mouse double minute (MDM)2-mediated p53 degradation (3). This is an auto-regulated, self-protective mechanism that prevents cells from malignant transformation. Notably, p53 can repress Myc activity by transcriptionally activating, for example, miR-145 which targets $M y c$ mRNA for translation silencing $(4,5)$, thus forming a negative feedback regulatory loop.

The most straightforward and effective strategy to treat cancer is to kill the cancerous cells. The commonly used anticancer drugs, such as cisplatin (6), actinomycin D (7) and adriamycin (8) are shown to inhibit tumor growth by promoting apoptosis. Recently, growing evidence has demonstrated that a number of natural products and derivatives from plants, particularly from medicinal plants used in traditional Chinese medicine (TCM), exhibit a tumor suppressive function by inducing the apoptosis of cancer cells and have potential for clinical application in cancer therapy (9). For instance, the natural anthraquinone emodin isolated from the TCM, Radix rhizome Rhei, can suppress the growth of numerous types of cancer cells $(10,11)$. Camptothecin, derived from the Chinese 'happy tree', Camptotheca acuminate, is a valuable natural product that inhibits the ligation of DNA following topo I-mediated strand breaks (12). In another retrospective population-based cohort study of a total of 729 patients with advanced breast cancer, it was suggested that TCM therapy can contribute to cancer treatment. Of this cohort, 115 patients were TCM users while 614 patients did not use TCM. The multivariate analysis demonstrated that, compared with non-users, the use of TCM was associated with a significantly decreased risk of all-cause mortality (13). All the aforementioned findings indicated that TCM is an important complementary and alternative medicine that can be used in the treatment of cancer.

Echinacoside $(\mathrm{ECH})$ is a phenylethanoid isolated from the stems of Cistanches salsa, a Chinese herbal medicine, which is an important crude drug used as an antisenium and antifatigue agent (14). It has been found that several phenylethanoids possess free radical scavenging properties and protect against oxidative stress-induced toxic injuries (15). In addition, more biological properties of ECH have since been elucidated. For example, ECH can rescue the increased levels of inflammatory cytokines and improve lung histopathological abnormalities in 
mice with acute lung injury $(16,17)$. Also, ECH has been shown to have protective effects on nerve tissue and improve behavioral disorders in murine models of Parkinson's disease (18). Notably, it has been found that ECH promotes cell proliferation and inhibits apoptosis in the mouse intestinal epithelial MODE-K cells (19). Thus far, however, less attention has been paid to the potential role of $\mathrm{ECH}$ in cancer prevention.

In this study, it was explored whether ECH treatment affects tumor cell growth and proliferation, and whether $\mathrm{ECH}$ induces apoptosis, elevates the production of reactive oxygen species (ROS) and reduces mitochondrial membrane potential (MMP), and consequently suppresses tumor cell growth. Furthermore the present study aimed to identify the molecular mechanisms responsible for ECH-mediated cell growth inhibition.

\section{Materials and methods}

Cell line, reagent and antibodies. SW1990 pancreatic adenocarcinoma cells (ATCC, Manassas, VA, USA) were cultured in Dulbecco's modified Eagle's medium supplemented with $10 \%$ fetal bovine serum, $50 \mathrm{U} / \mathrm{ml}$ penicillin and $0.1 \mathrm{mg} / \mathrm{ml}$ streptomycin at $37^{\circ} \mathrm{C}$ in a $5 \% \mathrm{CO}_{2}$ humidified atmosphere. $\mathrm{ECH}$ was purchased from Jrdun Biotechnology Corp. (Shanghai, China). Antibodies against AKT, P-AKT, ERK, P-ERK, JNK, p-JNK, P38, p-P38 and GAPDH were purchased from Cell Signaling Technology (Danvers, MA, USA); anti-Bax and anti-Bcl-2 were purchased from Santa Cruz Biotechnology Inc. (Santa Cruz, CA, USA); and anti-Caspase-3 was purchased from Abcam (Shanghai, China).

Cell viability assay. To assess the rate of tumor cell growth, Cell Counting kit-8 (CCK-8; Dojindo Molecular Technologies, Rockville, MD, USA) was used according to the manufacturer's instructions. Cell suspensions were seeded at 5,000 cells per well with ECH treatment for 0 , $12,24,48$ or $72 \mathrm{~h}$ in 96 -well culture plates. Cell growth inhibition was determined by adding WST- 8 reagent from the CCK- 8 kit at a final concentration of $10 \%$ to each well, and the absorbance of the samples was measured at $450 \mathrm{~nm}$ using a microplate reader (Multiskan MK3; Thermo Fisher Scientific, Inc., Waltham, MA, USA).

Hoechst 33342 staining. Cells (60\% confluence) were treated with Hoechst 33342 (Beyotime Institute of Biotechnology, Haimen, China) at a final concentration of $1 \mu \mathrm{g} / \mathrm{ml}$, incubated in $37^{\circ} \mathrm{C}$ incubator for $15 \mathrm{~min}$, washed with phosphate-buffered saline twice, fixed in $4 \%$ paraformaldehyde for $30 \mathrm{~min}$ at room temperature, and mounted onto slides. The morphological changes of the cell nuclei were observed under a fluorescence microscope (Olympus BX51, Melville, NY, USA). The normal nuclei were round and stained light blue, while the apoptotic nuclei were shrunken and stained bright blue.

Fluorescence-activated cell sorting (FACS) analyses. For the assessment of apoptosis, the fluorescein isothiocyanate (FITC)-Annexin V Apoptosis Detection kit (BD Biosciences, Shanghai, China) was used according to the manufacturer's instructions. Briefly, $5 \times 10^{4}$ cells were washed with ice-cold PBS, resuspended in $0.1 \mathrm{ml}$ binding buffer (Beyotime Institute

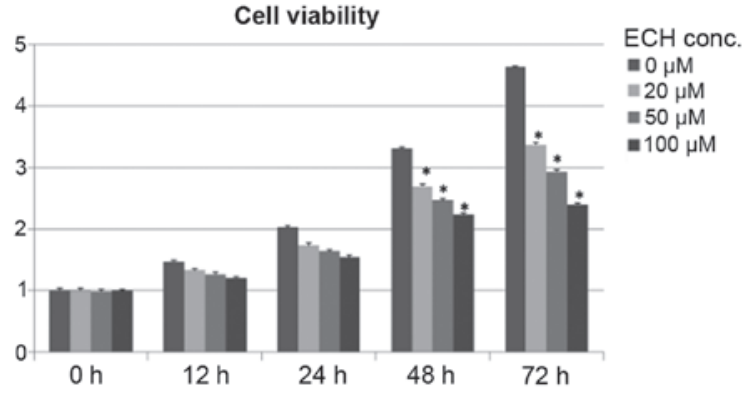

Figure 1. ECH treatment inhibits SW1990 cell proliferation. Cell suspensions were seeded at 5,000 cells per well in 96-well culture plates and treated with different doses of ECH for 0, 12, 24, 48 or $72 \mathrm{~h}$. The rate of cell growth was determined by Cell Counting kit- 8 ( $\mathrm{P}<0.05$, compared with the control). $\mathrm{ECH}$, echinacoside.

of Biotechnology), and stained with $10 \mathrm{ml}$ of FITC-conjugated Annexin $\mathrm{V}(10 \mathrm{mg} / \mathrm{ml})$ and $10 \mathrm{ml}$ propidium iodide (PI) $(50 \mathrm{mg} / \mathrm{ml})$. Following incubation for $15 \mathrm{~min}$ at room temperature in the dark and addition of $400 \mathrm{ml}$ binding buffer, the cells were analyzed by a flow cytometer (C6; BD Biosciences, Shanghai, China).

Measurement of reactive oxygen species (ROS). To evaluate the production of ROS, the Reactive Oxygen Species Assay kit (Vigorous Biotechnology, Beijing, China) was used according to the manufacturer's instructions. Briefly, cells $(80 \%$ confluence) were harvested and washed with PBS prior to staining with the dihydroethidium (DHE) solution (Beyotime Institute of Biotechnology). Cells were then analyzed by flow cytometric analyses.

Measurement of mitochondrial membrane potential (MMP). A tetramethylrhodamine methyl ester (TMRM) Assay kit (ImmunoChemistry Technologies, Bloomington, MN, USA) was used to detect the changes in MMP. Briefly, cells (80\% confluence) were harvested, washed with PBS and stained with TMRM for $15-20 \mathrm{~min}$ in a $37^{\circ} \mathrm{C}$ incubator. Cells were then washed once with PBS and subjected to flow cytometric analyses.

Immunoblotting analyses. The cells (80\% confluence) were harvested and lysed in RIPA buffer (Jrdun Biotechnology) consisting of $50 \mathrm{mM}$ Tris- $\mathrm{HCl}, \mathrm{pH} 7.4 ; 150 \mathrm{mM} \mathrm{NaCl}, 1 \mathrm{mM}$ EDTA, $1 \%$ NP-40, $1 \%$ sodium deoxycholic acid and $0.1 \%$ SDS and freshly added proteasome inhibitors. Equal quantities of clear cell lysate were used for immunoblotting analysis as described previously (20).

Statistical analysis. Quantitative data are expressed as the mean \pm standard deviation. Statistical differences were evaluated by unpaired Student's t-test using statistical SPSS 15.0 software. $\mathrm{P}<0.05$ was considered to indicate a statistically significant difference.

\section{Results}

ECH suppresses tumor cell growth. Although it has been reported that ECH exhibits a protective role by inhibiting 
A

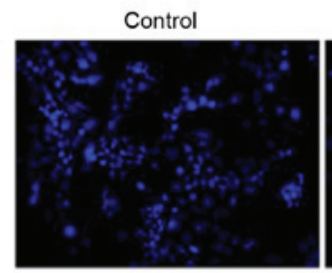

B

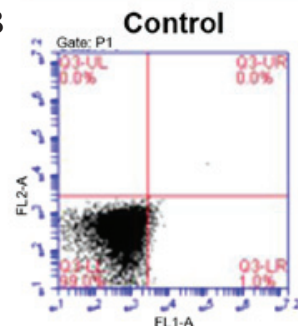

$\mathrm{ECH}, 50 \mu \mathrm{M}$

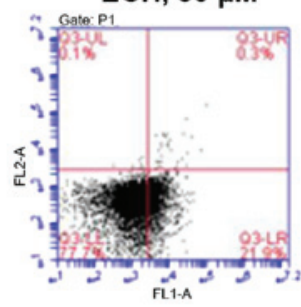

$\mathrm{ECH}, 20 \mu \mathrm{M}$

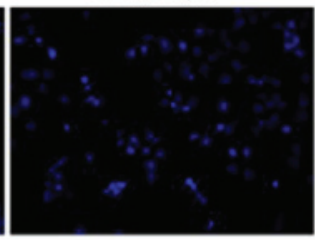

$\mathrm{ECH}, 20 \mu \mathrm{M}$

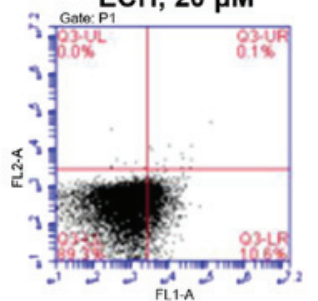

$\mathrm{ECH}, 100 \mu \mathrm{M}$

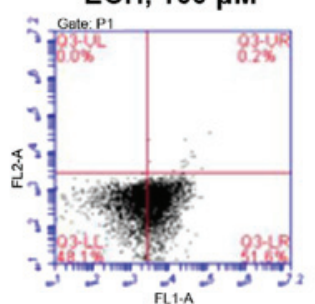

$\mathrm{ECH}, 50 \mu \mathrm{M}$

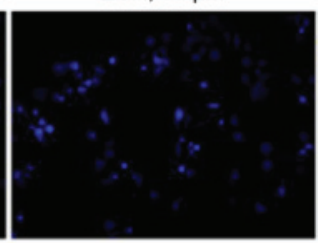

C
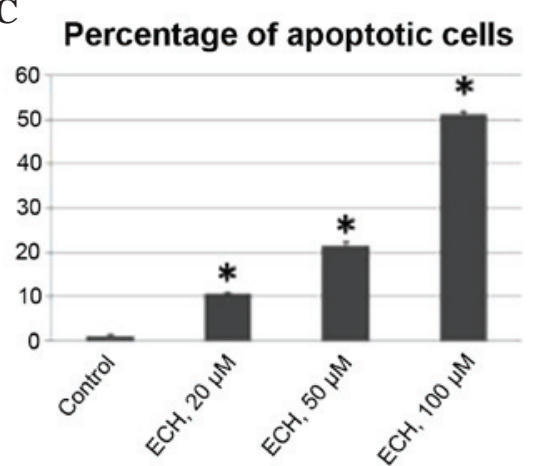

Figure 2. ECH treatment induces apoptosis in SW1990 cells. (A) ECH induces apoptosis in a dose-dependent manner as determined by Hoechst 33342 staining (magnification, $\mathrm{x} 400$ ). (B) ECH induces apoptosis in a dose-dependent manner as determined by fluorescence-activated cell sorting analyses. (C) Quantification of apoptotic cells in (B). ( $\mathrm{P}<0.001$, compared with the control). ECH, echinacoside.

\section{A}
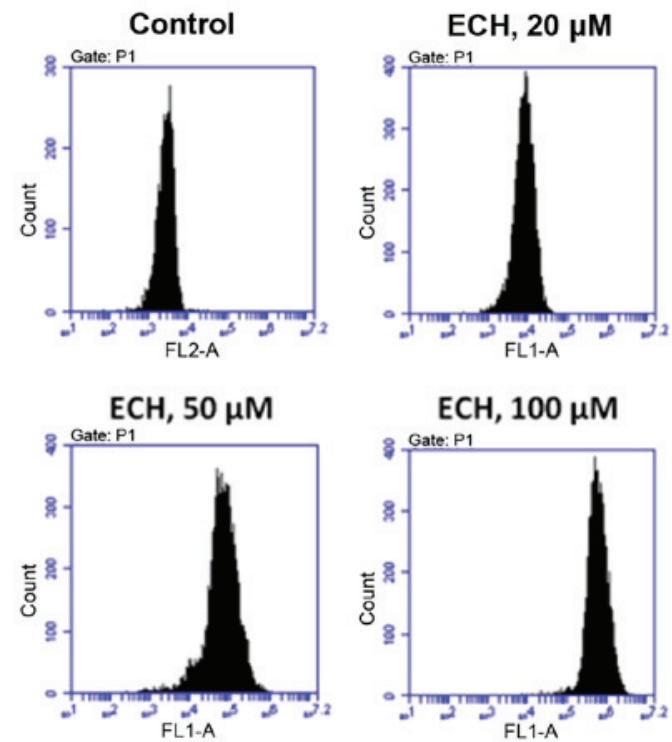

B Fluorescence intensity

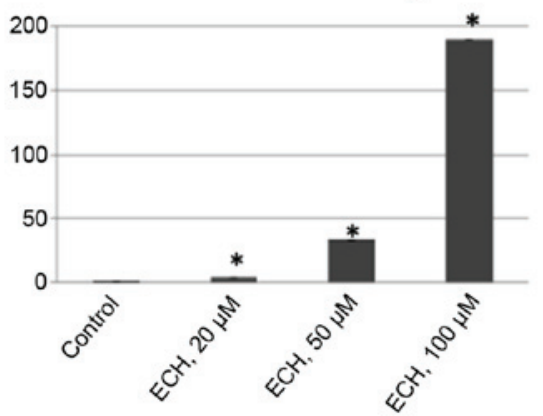

Figure 3. ECH promotes ROS production in SW1990 cells. (A) Cells were treated with different doses of ECH, and ROS production was assessed by the Reactive Oxygen Species Assay kit. (B) Quantification of fluorescence intensity in (A). ( $\mathrm{P}<0.001$, compared with control). ECH, echinacoside; ROS, reactive oxygen species.

apoptosis and inflammatory signals in somatic cells, such as neuronal and intestinal epithelial cells (16-19), it remains elusive whether ECH controls cancerous cell growth and proliferation. To test this, a cell survival assay was conducted by treating SW1990 cells, derived from a grade II pancreatic adenocarcinoma, with titrated doses of ECH as shown in Fig. 1. Notably, it was demonstrated that ECH significantly retards tumor cell proliferation in a dose-dependent manner during a 5-day culture period (Fig. 1).

ECH triggers apoptosis. As loss of apoptosis is one of the major causative mechanisms underlying the uncontrolled proliferation of pancreatic cancer cells (21), the present study performed a set of experiments to determine whether $\mathrm{ECH}$ 
A
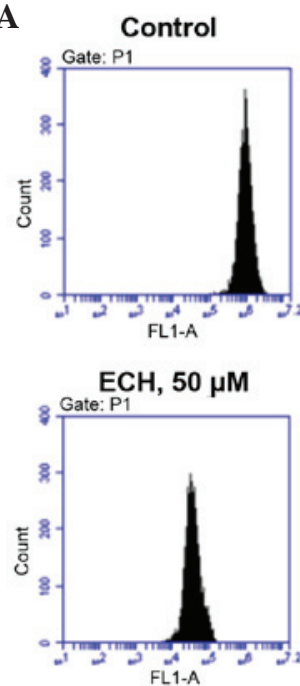

$\mathrm{ECH}, 20 \mu \mathrm{M}$

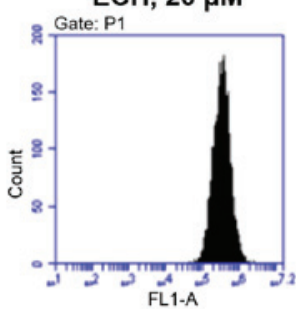

$\mathrm{ECH}, 100 \mu \mathrm{M}$

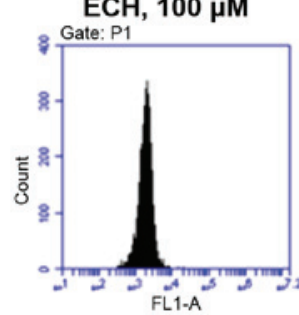

B Fluorescence intensity

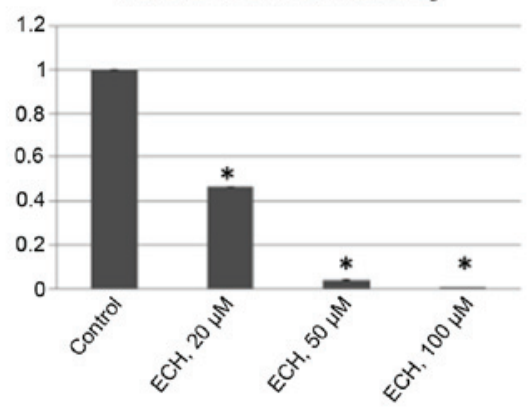

Figure 4. ECH reduces MMP in SW1990 cells. (A) Cells were treated with different doses of ECH and MMP was assessed by a TMRM Assay kit. (B) Quantification of fluorescence intensity in (A). ("P<0.001, compared with control). ECH, echinacoside; MMP, mitochondrial membrane potential; TMRM, tetramethylrhodamine methyl ester.

A
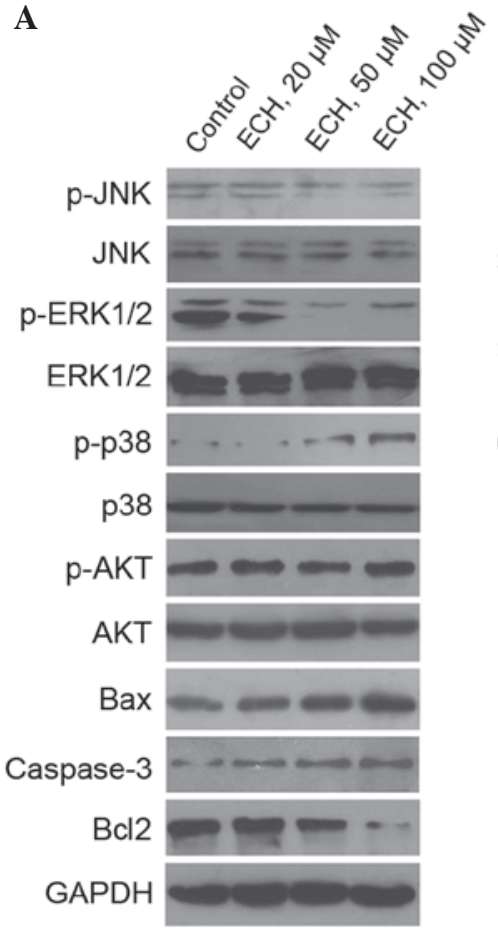

B

Relative protein expression compared to GAPDH

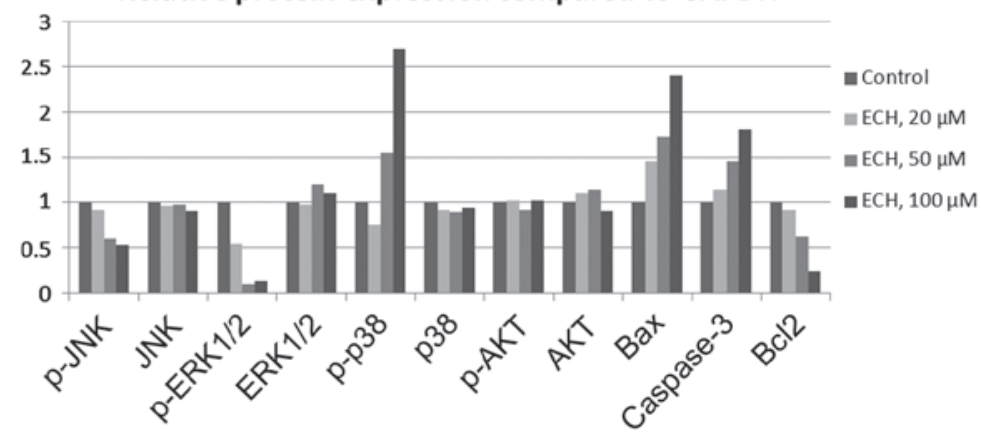

Figure 5. ECH regulates mitogen-activated protein kinase signaling pathways and apoptosis-associated proteins. (A) Cells treated with different doses of $\mathrm{ECH}$ were harvested and subjected to immunoblotting analyses using antibodies as indicated. (B) Quantification of immunoblotting results of (A). ECH, echinacoside; JNK, NH2-terminal kinase; ERK, extracellular-signal regulated kinases.

triggers apoptosis. Firstly, by staining the nuclei of tumor cells with Hoechst 33342, it was demonstrated that ECH results in apoptosis in a dose-dependent manner (Fig. 2A). Additionally, FACS analyses was conducted using Annexin V/PI staining to further confirm the apoptotic effect of ty ECH (Fig. 2B). The average percentage of apoptotic cells was $1.1 \%$ under normal culture conditions, while this percentage significantly increased to $10.6,21.4$ and $51.3 \%$ in response to $\mathrm{ECH}$ treatment in a dose-dependent manner (Fig. 2C). These results, together with the cell viability assay shown in Fig. 1, demonstrate that
ECH treatment suppresses the proliferation of tumor cells by triggering apoptosis.

ECH prompts the production of reactive oxygen species (ROS). Several cancer chemotherapeutic drugs, such as doxorubicin and cisplatin, have been shown to be potent generators of ROS which are crucial role in apoptosis induction under physiological and pathological conditions (22). Thus, it was investigated whether ECH treatment could also regulate ROS production. To test this, DHE, which can be oxidized to 
generate ethidium that intercalates with DNA, was used in this study to evaluate ROS production. It was found that, like other anticancer drugs, ECH also stimulates ROS production in a dose-dependent manner as shown by the elevated fluorescence intensity upon ECH treatment (Fig. 3).

ECH reduces MMP. Mitochondrial dysfunction has been shown to be implicated in the induction of apoptosis. Opening of the mitochondrial permeability transition pore has been demonstrated to induce the depolarization of the transmembrane potential and the release of pro-apoptotic factors (23). Therefore, it was tested whether ECH could induce loss of MMP in tumor cells by performing a TMRM assay, which is a well-established approach, as the intensity of TMRM fluorescence is proportional to the membrane potential. It was demonstrated that ECH treatment significantly reduces MMP in a dose-dependent manner (Fig. 4).

ECH controls tumor cell growth via mitogen-activated protein kinase (MAPK), but not AKT, signals. To further investigate the molecular basis of ECH-mediated tumor cell death, the activity of several vital signaling pathways, such as MAPK and AKT $(24,25)$, which control cell survival and death was examined. The MAPKs are evolutionarily conserved, proline-directed Ser/Thr protein kinases, including extracellular signal-regulated kinases (ERKs), c-Jun $\mathrm{NH}_{2}$-terminal kinase (JNKs) and the p38 family members which are activated through three-tier kinase signaling cascades $(26,27)$. In this study, the expression of MAPKs and AKT, as well as their activated phosphorylated forms, was assessed and it was revealed that ECH markedly suppresses JNK and ERK1/2 activity, but enhances p38 activity (Fig. 5). Notably, it was demonstrated that AKT activity, which is also critically important for cell proliferation, is not affected by ECH treatment (Fig. 5). In addition, it was shown that ECH treatment elevates the expression of Bax and Caspase- 3 whilst reduces Bcl-2 expression (Fig. 5), which is consistent with Fig. 2. Thus, the results indicate that $\mathrm{ECH}$ triggers tumor cell apoptosis via the MAPK pathway.

\section{Discussion}

To the best of our knowledge, this is the first study to show that $\mathrm{ECH}$ has a tumor suppressive function by triggering apoptosis (Fig. 2), promoting ROS production (Fig. 3) and inducing mitochondrial membrane potential depolarization (Fig. 4), consequently leading to tumor cell growth inhibition (Fig. 1). Furthermore, the molecular basis of ECH-mediated tumor cell death was shown to occur by regulating MAPK signaling pathways (Fig. 5). These findings demonstrate a novel function of ECH in preventing tumorigenesis and thus suggest that it may be a candidate agent for cancer therapy.

The majority of anticancer drugs can induce tumor cell apoptosis, senescence and/or cell cycle arrest, which leads to inhibition of tumor cell growth and proliferation. Cell cycle arrest is a cellular response to mild stress signals that allow cells to repair damaged DNA prior to initiating replicative DNA synthesis or mitosis, whereas apoptosis and senescence (permanent cell cycle arrest) occur in response to stress signals that eliminate irreparable or malignant cells $(28,29)$. Therefore, only the apoptotic effect of ECH on tumor cells was assessed in this study, as killing cancerous cells is a major criterion for evaluating the potency of an anticancer agent. Notably, it was demonstrated that $\mathrm{ECH}$ induces the expression of Bax (Fig. 5), a pro-apoptotic gene, transcriptionally activated by the tumor suppressor p53 (30). Thus, it is worthwhile to test whether ECH can activate the p53 signaling pathway. If so, ECH may also be able to elicit p53-dependent cell cycle arrest, senescence, apoptosis or autophagy. In this study, the tumor suppressive function of ECH in the SW1990 pancreatic adenocarcinoma cell line was elucidated. However, further studies observing more pancreatic cancer cell lines, are required. It has been shown that mutations in the oncogenic protein RAS and the tumor suppressor p53 are associated with the development of pancreatic cancer (31); however, the SW1990 cell line does not harbor any p53 mutation, according to the IARC p53 database (http://p53.iarc.fr/CellLines.aspx). Hence, it is important to investigate whether ECH can affect the growth and proliferation of other pancreatic cancer cell lines with different p53 mutations. Additionally, it would be interesting to determine whether $\mathrm{ECH}$ is able to promote apoptosis and inhibit the growth of other types of tumors.

ROS elevation and MMP reduction, which were caused by ECH treatment, have been shown to be essential in apoptosis induction (22). In addition, it has been found that ROS can induce the oxidation of the mitochondrial pores contributing to the release of cytochrome $c$, an intermediate in apoptosis, due to disruption of the MMP (22). Thus, it remains to be determined whether ECH disrupts MMP indirectly through induction of ROS. Moreover, ROS-elicited oxidative stress has also been shown to be involved in modulating a myriad of cell-growth controlling signals, including p53, NF- $\kappa \mathrm{B}$, HIFs and PI3K (32). It remains to be determined whether and, if so, how ECH regulates these important signaling pathways. Notably, oxidative stress causes various neurodegenerative diseases due to the high oxygen consumption, weak antioxidative systems and terminal-differentiation characteristics of the central nervous system (33). However, several studies have shown that $\mathrm{ECH}$ has protective and anti-apoptotic effects on nervous tissue. In this regard, it is reasonable to hypothesize that $\mathrm{ECH}$ may reduce ROS production in the terminal-differentiated neural cells. Therefore, it also remains to be determined whether the regulation of ROS production by $\mathrm{ECH}$ is dependent on the cell differentiation status. Hence, the present results together with other studies reveal that $\mathrm{ECH}$, the widely used TCM, can be an important chemotherapeutic strategy not only for the treatment of neurodegenerative diseases but also malignant carcinomas.

Recently, using TCM in cancer therapy has gained increasingly more attention. The potential of natural products from medicinal plants used in TCM has been recognized by the scientific community even in the western world (9). Efforts are required to elucidate the underlying mechanisms of action of these natural products, which may eventually lead to the development of efficient and safe medicines for cancer therapy.

In conclusion, the present study demonstrated the tumor inhibitory function of $\mathrm{ECH}$ and also elaborates the molecular basis of ECH-mediated tumor suppression, thus suggesting the potential clinical application of ECH in cancer therapy. 


\section{Acknowledgements}

This study was funded and supported by the Key Program of Scientific Research of FMU(grant no. 09ZD014). The authors would like to thank Biomedworld (Shanghai, China) for help with editing the manuscript.

\section{References}

1. Hanahan D and Weinberg RA: Hallmarks of cancer: The next generation. Cell 144: 646-674, 2011.

2. Alderton GK: Transcription: The transcriptional effects of MYC. Nat Rev Cancer 14: 513, 2014.

3. Zindy F, Eischen CM, Randle DH, Kamijo T, Cleveland JL, Sherr CJ and Roussel MF: Myc signaling via the ARF tumor suppressor regulates p53-dependent apoptosis and immortalization. Genes Dev 12: 2424-2433, 1998.

4. Sachdeva M, Zhu S, Wu F, Wu H, Walia V, Kumar S, Elble R, Watabe $\mathrm{K}$ and Mo YY: p53 represses c-Myc through induction of the tumor suppressor miR-145. Proc Natl Acad Sci USA 106 3207-3212, 2009

5. Liao JM, Cao B, Zhou X and Lu H: New insights into p53 functions through its target microRNAs. J Mol Cell Biol 6 : 206-213, 2014

6. Zamble DB and Lippard SJ: Cisplatin and DNA repair in cancer chemotherapy. Trends Biochem Sci 20: 435-439, 1995.

7. Kleeff J, Kornmann M, Sawhney H and Korc M: Actinomycin D induces apoptosis and inhibits growth of pancreatic cancer cells. Int J Cancer 86: 399-407, 2000.

8. Tacar O, Sriamornsak P and Dass CR: Doxorubicin: An update on anticancer molecular action, toxicity and novel drug delivery systems. J Pharm Pharmacol 65: 157-170, 2013.

9. Efferth T, Li PC, Konkimalla VS and Kaina B: From traditional Chinese medicine to rational cancer therapy. Trends Mol Med 13 353-361, 2007.

10. Yim H, Lee $\mathrm{YH}$, Lee $\mathrm{CH}$ and Lee SK: Emodin, an anthraquinone derivative isolated from the rhizomes of Rheum palmatum, selectively inhibits the activity of casein kinase II as a competitive inhibitor. Planta Med 65: 9-13, 1999.

11. Zhang L, Chang CJ, Bacus SS and Hung MC: Suppressed transformation and induced differentiation of HER-2/neu-overexpressing breast cancer cells by emodin. Cancer Res 55: 3890-3896, 1995.

12. Pommier Y: Topoisomerase I inhibitors: Camptothecins and beyond. Nat Rev Cancer 6: 789-802, 2006.

13. Lee YW, Chen TL, Shih YR, Tsai CL, Chang CC, Liang HH, Tseng SH, Chien SC and Wang CC: Adjunctive traditional Chinese medicine therapy improves survival in patients with advanced breast cancer: A population-based study. Cancer 120: 1338-1344, 2014.

14. Xiong Q, Hase K, Tezuka Y, Namba T and Kadota S: Acteoside inhibits apoptosis in D-galactosamine and lipopolysaccharide-induced liver injury. Life Sci 65: 421-430, 1999.
15. Gao JJ, Igalashi K and Nukina M: Radical scavenging activity of phenylpropanoid glycosides in Caryopteris incana. Biosci Biotechnol Biochem 63: 983-988, 1999.

16. Zhang Y, Xing J, Ai T, Wen T, Guan L and Zhao J: Protection of echinacoside against acute lung injury caused by oleic acid in rats. Free Radic Res 41: 798-805, 2007.

17. Li X, Gou C, Yang H, Qiu J, Gu T and Wen T: Echinacoside ameliorates D-galactosamine plus lipopolysaccharide-induced acute liver injury in mice via inhibition of apoptosis and inflammation. Scand J Gastroenterol 49: 993-1000, 2014.

18. Zhao Q, Gao J, Li W and Cai D: Neurotrophic and neurorescue effects of Echinacoside in the subacute MPTP mouse model of Parkinson's disease. Brain Res 1346: 224-236, 2010.

19. Jia Y, Guan Q, Guo Y and Du C: Echinacoside stimulates cell proliferation and prevents cell apoptosis in intestinal epithelial MODE-K cells by up-regulation of transforming growth factor- $\beta 1$ expression. J Pharmacol Sci 118: 99-108, 2012.

20. Liao P, Wang W, Shen M, Pan W, Zhang K, Wang R, Chen T, Chen Y, Chen $\mathrm{H}$ and Wang P: A positive feedback loop between EBP2 and c-Myc regulates rDNA transcription, cell proliferation and tumorigenesis. Cell Death Dis 5: e1032, 2014.

21. Westphal S and Kalthoff H: Apoptosis: Targets in pancreatic cancer. Mol Cancer 2: 6, 2003.

22. Simon HU, Haj-Yehia A and Levi-Schaffer F: Role of reactive oxygen species (ROS) in apoptosis induction. Apoptosis 5: 415-418, 2000.

23. Ly JD, Grubb DR and Lawen A: The mitochondrial membrane potential (deltapsi(m)) in apoptosis; an update. Apoptosis 8: 115-128, 2003.

24. Munshi A and Ramesh R: Mitogen-activated protein kinases and their role in radiation response. Genes Cancer 4: 401-408, 2013.

25. Abraham AG and O'Neill E: PI3K/Akt-mediated regulation of p53 in cancer. Biochem Soc Trans 42: 798-803, 2014.

26. Tournier C: The 2 Faces of JNK Signaling in Cancer. Genes Cancer 4: 397-400, 2013.

27. Koul HK, Pal M and Koul S: Role of p38 MAP kinase signal transduction in solid tumors. Genes Cancer 4: 342-359, 2013.

28. Brady CA and Attardi LD: p53 at a glance. J Cell Sci 123: 2527-2532, 2010

29. Carvajal LA and Manfredi JJ: Another fork in the road-life or death decisions by the tumour suppressor p53. EMBO Rep 14: 414-421, 2013.

30. Miyashita T and Reed JC: Tumor suppressor p53 is a direct transcriptional activator of the human bax gene. Cell 80: 293-299, 1995.

31. Morton JP, Timpson P, Karim SA, Ridgway RA, Athineos D, Doyle B, Jamieson NB, Oien KA, Lowy AM, Brunton VG, et al: Mutant p53 drives metastasis and overcomes growth arrest/senescence in pancreatic cancer. Proc Natl Acad Sci USA 107: 246-251, 2010.

32. Schieber $M$ and Chandel NS: ROS function in redox signaling and oxidative stress. Curr Biol 24: R453-R462, 2014.

33. Li J, O W, Li W, Jiang ZG and Ghanbari HA: Oxidative stress and neurodegenerative disorders. Int J Mol Sci 14: 24438-24475, 2013. 